\title{
De Quervain's tenosynovitis: a non-randomized two-armed study comparing ultrasound-guided steroid injection with surgical release
}

\author{
A. K. Bhat $\cdot$ R. Vyas $\cdot$ A. M. Acharya ${ }^{1}(1) \cdot$ K. V. Rajagopal ${ }^{2}$
}

Received: 28 September 2020 / Accepted: 29 January 2022 / Published online: 23 February 2022

(c) The Author(s) 2022

\begin{abstract}
Purpose Ultrasonography is currently used for both diagnostic and therapeutic purposes in de Quervain's tenosynovitis. There is a dearth of information on how effective an ultrasound-guided (USG) steroid injection is when compared to surgical release of the first extensor compartment. Hence, we performed a non-randomized two-armed comparison study to test our hypothesis that USG guided steroid injection is equally effective as surgery.

Method 62 consecutive patients participated in the study with 32 of them selecting the option of USG guided injection (Set A), and the rest undergoing surgical release (Set B). We reviewed them after 3 and 6 weeks and 6 months for functional outcome using DASH, PRWE and VAS scores, recurrence, or any complications. They were further followed if they were symptomatic.

Results The DASH/PRWE/VAS scores improved at the end of 6 months from 81.7/79.3/6.8 to 1.0/1.7/1.0, respectively for patients undergoing USG guided steroid injection. Similarly, for the patient undergoing surgery, the scores improved from 82.2/81.5/6.7 to $1.7 / 3.4 / 1.0$, respectively. This was statistically significant in both the groups $(p<0.05)$ and was comparable to each other. Two patients in Set A came back with recurrence at eight and 10 months and two reported occasional pain on heavy work. Three patients had tenderness and two had numbness in Set B at the scar site.

Conclusion We observed that USG guided steroid injections are comparable to surgical release in terms of pain relief, functional outcome, complications.
\end{abstract}

Keywords De Quervain's tenosynovitis · Steroid injection · Ultrasonography · First extensor compartment · Surgical release

\section{Introduction}

De Quervain's tenosynovitis (DQV) is primarily a painful tendinosis where tenderness and soreness are felt at the region of the radial styloid while performing activities, which involve wrist and thumb movements [1]. It occurs due to repetitive activities, common in women in the age group of 30-50 years [1-4]. Some authors attribute the condition to be due to inflammatory involvement of the synovial sheath

A. M. Acharya

anmacharya@gmail.com

1 Division of Hand and Microsurgery, Department of Orthopaedics, Kasturba Medical College, Manipal Academy of Higher Education, Manipal 576104, India

2 Department of Radiodiagnosis, Kasturba Medical College, Manipal Academy of Higher Education, Manipal, 576104, India inside the first dorsal compartment (FDC) which surrounds the abductor pollicis longus (APL) and the extensor pollicis brevis (EPB) tendons. Samples collected from affected patients have shown the presence of neutrophil elastase and cyclooxygenase which indicates the presence of inflammation and hence the damaging effects on the collagen structure in the FDC [5]. However, other studies have objected to the term 'tenosynovitis' in DQV, as no inflammation could be demonstrated. The tendinosis is more of myxoid degeneration rather than acute inflammation that is induced due to mechanical impingement of the Abductor pollicis longus (APL) and extensor pollicis brevis (EPB) tendons in the tight fibro-osseous canal, which stimulates the nociceptors [1-4]. The thickening of the synovial sheath may result in entrapment of the tendon in the tight canal during an excursion of the APL and EPB tendons [1-5]. There is increased friction between the contents of the canal, which could be the source of pain and tenderness. [1-6]. Anatomical variations in the 
first extensor compartment such as the presence of a separate septum for EPL and multiple slips of APL are also said to be associated with De Quervain's tenosynovitis [5-7].

Patients undergo a spectrum of treatment including nonsurgical measures of rest, splinting, casting, non-steroidal anti-inflammatory drugs (NSAIDs), and local steroid injections [1]. If the above measures fail, surgical treatment remains the only option [1]. However, failure of treatment and recurrence have been described in both non-operative and surgical modalities due to missed or incomplete release of sub-compartment for EPB in the majority of studies [1, 3, 5-9].

Non-surgical management other than steroids has shown limited benefits and a high rate of relapse $[1,6,10,11]$. Steroid injections are a widely accepted and its efficacy has been proved in randomized control trials (RCT) and systematic reviews $[1,4,12,13]$. However, with blind injections, the recurrence rates from the literature have varied from nil to as high as $52 \%$ [1]. This is attributed to be due to septation of the first extensor compartment where the blind injections can miss one of the sub-compartments and lead to inadequate relief of symptoms as shown in cadaveric and clinical studies $[14,15]$. The use of steroids has also shown complications like injury and rupture of APL, EPB and dorsal sensory branches of radial nerve apart from flare, hypopigmentation and atrophy of the skin [16-18]. The introduction of ultrasonography (USG) guided steroid injections has brought in a new paradigm as it can visualize the presence of a sub-compartment and allow for more accurate placement of the needle for steroid injection [19,20]. The success of USG guided steroid injections as shown in some of the reports in the last decade introduces it as a potential primary mode of treatment for de Quervain's tenosynovitis [19-26]. However, it remains to be seen whether it can produce an equally better result when compared to a carefully performed surgical release where excellent and predictable long-term results have been reported [27, 28]. There are no studies comparing these two modalities of treatment. According to our hypothesis, both treatments are comparable for the treatment of de Quervain's tenosynovitis. Associated complications of surgery can be avoided if USG guided steroid injection was found to have equal outcomes.

\section{Materials and methods}

This was a non-randomized two-arm study conducted after institutional ethical board clearance. The aim of the study was to compare the functional outcome, recurrence and complications comparing USG guided steroid injection with surgical release of the first extensor compartment. Consecutive patients with clinically diagnosed de Quervain's tenosynovitis were selected as defined by radial sided wrist pain, radial-styloid tenderness, a positive Finkelstein's test, and those who did not improve after a trial of conservative management of a minimum of 4 weeks were included in the study. Patients who had taken prior treatment with multiple steroid injections, those who had a contraindication to steroid therapy, those with causes like past fractures/ dislocations, inflammatory disorders or previous surgery at the same region, $\mathrm{CMC}$ or wrist arthritis or neoplasm which would have altered the functional outcomes were excluded (Table 1).

62 consecutive patients were grouped into Set A (USG guided injection-32) and Set B (Surgical release-30). The demographic characteristics of these two groups were comparable. (Table 1). Patients were allotted based on shared decision-making of their choices after explaining both the procedures in detail and discussion with the operating surgeon. All patients underwent pre-operative USG examination to aid in confirming the diagnosis of tenosynovitis, and more importantly, to note the presence of anatomical variations. This information would aid both the operating surgeon and the radiologist during their intervention procedures. Qualified radiologists from the department of radiology with more than 5 years of experience in musculoskeletal ultrasonography did the USG examinations.

A pilot study was done initially in six patients to standardize the technique of USG guided injection. Transverse and longitudinal approaches were used, and the former was found to be better, as it was easy to access both the compartments and avoid the sensory branches of the radial nerve (Fig. 1). The injections were given by a single dedicated musculoskeletal radiologist (KVR) with more than 15 years experience to ensure the uniformity of technique.

\section{Injection technique}

Under aseptic conditions, a Philips IU 22 USG machine and $12.3 \mathrm{~Hz}$ linear probe were used to visualize cross-sectional anatomy of the first extensor compartment. A 20-gauge needle was used to inject $2 \mathrm{cc}$ of $2 \%$ lignocaine and $20 \mathrm{mg}$ methylprednisolone acetate $(20 \mathrm{mg} / \mathrm{ml})$ into the first extensor compartment of the wrist around the APL and EPB tendons.

If a separate compartment was noted for EPB, the injection was split equally for both the compartments with similar infiltration techniques under USG guidance (Fig. 1b). Special care was taken to avoid the sensory branches of the radial nerve. Patients undergoing injections were advised a thumb spica splint and light work for 3 weeks followed by resumption of their routine activities.

\section{Surgical technique}

A single-hand surgeon performed all surgical releases. A transverse incision was made and decompression of the 
Table 1 Demographic data of the two sets of patients

\begin{tabular}{llll}
\hline Parameter & & Patient details & \\
\cline { 4 - 4 } & & $\begin{array}{l}\text { USG guided injec- } \\
\text { tion }\end{array}$ & Surgical release \\
\hline Gender & Female/total & $26 / 32$ & $21 / 30$ \\
Mean age & In years & 46.17 & 49.13 \\
Occupation: number & Labourer & 3 & 4 \\
& Homemaker & 20 & 20 \\
& Student & 2 & 0 \\
Desk job & 6 & 4 \\
Right hand involvement & Nurse & 1 & 2 \\
Duration of symptoms & & $20 / 32$ & $18 / 30$ \\
Mean (for total patients) & Right hand/total & $23 / 32$ & $22 / 30$ \\
23 weeks( 4-80 weeks) & & & \\
& & & 14 \\
Patients with comorbidities & Upto 3 months & 9 & 13 \\
Patients with history of injury & Upto 6 months & 2 & 2 \\
Separate compartment for EPB & Upto 9 months & 4 & 1 \\
Multipennate APL & Upto 12 months & 4 & 8 \\
\hline & & $7 / 32$ & $6 / 30$ \\
& Multipennate/total & $29 / 32$ & $16 / 30$ \\
& & &
\end{tabular}
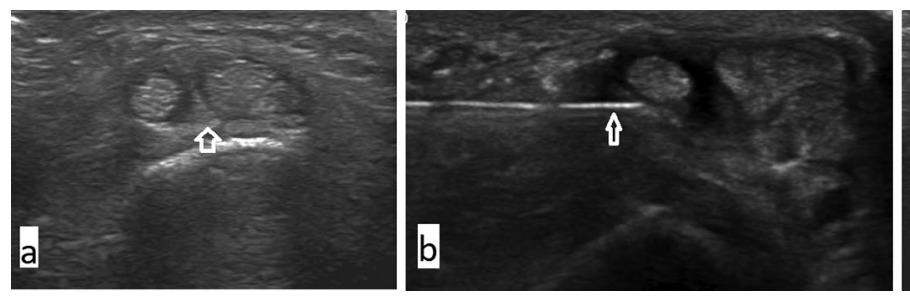

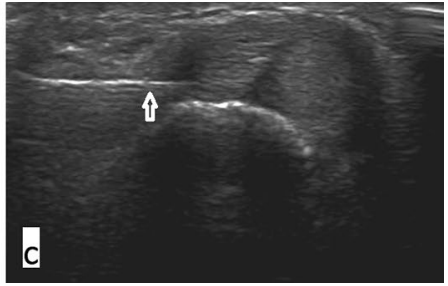

compartment due to the presence of a septum in between. c Arrow showing the needle in the first dorsal compartment. Note that the infiltrate has evenly spread around both the tendons

sal compartment. b Arrow showing the needle in the EPB compartment with infiltrating. Note that the infiltrate has not reached the APL

first extensor compartment was completed, ensuring the release of any sub-compartment and checking the excursion of EPB and APL separately. They were immobilized in a thumb spica slab for 3 days and later mobilized in a thumb spica splint for a further 3 weeks. This was followed by the resumption of their activities.

We reviewed all patients after 3 and 6 weeks and 6 months for the functional outcome, recurrence, or any complications. We used the disabilities of arm, shoulder and hand (DASH) score, patient rated wrist evaluation (PRWE) score, and visual analogue score (VAS) $[13,16]$. The 30 -item selfreported DASH questionnaire looks at the patient's ability to perform specific upper extremity activities and difficulty and interference with daily life. The score is documented on a 5-point Likert scale. PRWE is a 15-item questionnaire used for specific wrist problems and was developed to assess wrist pain and disability in activities of daily living. It has two subscales of pain and function and each of them has an item (five for pain and 10 for function) rated from 1 to 10 . The maximum score for each of these two sections is 50 and the minimum is 0 . The VAS is a widely used unidimensional measure of pain intensity. We followed a simple method in using a straight horizontal line of $10 \mathrm{~cm}$ orientated from the left (worst - maximum pain at $10 \mathrm{~cm}$ mark) to the right (best - no pain at zero mark). The patient marks the point on the line, which they perceive to be their current state of pain at the site of pathology. The lesser the score suggests a better outcome in all the three scores.

We chose 6 months as this was the maximum likely time gap after which recurrence was unlikely to be reported [10, 29]. However, patients who returned back after 6 months were also assessed based on their symptoms and signs and 
their status documented. Patients who did not improve after the single steroid injection and had a relapse of symptoms was planned to be treated surgically. Relapse rate and intraoperative findings were to be recorded to establish etiology.

\section{Statistical methodology}

Mean scores were calculated for DASH, PRWE and VAS scores at every visit and were compared using repeated ANOVA measures. Total change in percentage was used to compare scores at each follow-up. Relapse rates and changes in treatment were reported. $P$-value $<0.05$ was considered statistically significant.

\section{Results}

Table 1 shows the demographic characteristics of both sets of patients. The majority of them were females and homemakers in their 4th decade. Among the 62 patients, $82.2 \%$ were middle-aged women with a mean age of 46.1 years involved in household activities. Most of our patients were right-handed $(72 \%)$ having symptoms on the dominant hand $(61 \%)$. (Table 1) The mean duration of symptoms was 23 weeks (4-80 weeks). About $80 \%$ of the patients did not have any associated comorbidities. On pre-op USG evaluation, 48 patients (77.4\%) had multipennate APL, 34 patients (54.8\%) had sub-compartment for EPB and one patient was found to have absent EPB (Hiranuma type 4) [21].

\section{Functional outcome}

The mean DASH, PRWE and VAS scores of patients in both the groups improved marginally following the initial conservative management of 4 weeks, which was found to be insignificant (Table 2).

At 6 weeks, both the group of patients showed significant improvement (Table 2). The DASH/PRWE/VAS scores improved from $82.2 / 81.5 / 6.7$ to $1.7 / 3.4 / 1.0$, respectively for patients undergoing surgery (Table 2). Similarly, for a patient undergoing USG guided steroid injection, the scores improved from $81.7 / 79.3 / 6.8$ to $1.0 / 1.7 / 1.0$, respectively
(Table 2). At the end of 6 months, there was a statistically significant difference in both the groups $(p<0.05)$ and were comparable to each other.

\section{Recurrence}

We did not observe recurrence in either group at the end of 6 months of follow-up. Twelve patients came for follow-up after 6 months out of which seven had received USG guided steroid injections. Those patients had an average follow-up of 14.5 months (12-24 months). Two patients who had received USG guided steroid injections showed recurrence, which was observed at eight and 10 months, respectively after the index injection. Both of them incidentally had a single compartment for EPB tendon on USG evaluation. One of them was a 35-years-old computer software professional who presented 8 months after the injection. She had symptoms for 4 weeks prior to the presentation. Another patient was a 59-year-old homemaker who had a history of soft tissue trauma involving the wrist 8 months after the index injection. She presented to us 2 months later with symptoms of recurrence. Both patients were unwilling for surgery and hence continued on medications and splinting.

\section{Complications}

Apart from two patients with recurrence, two other patients reported pain only on heavy work and the rest had complete relief of symptoms and returned to their daily activities.

Out of those 30 patients who underwent surgical release, three had scar tenderness, which resolved by the 6th month follow-up. Two patients have decreased sensations over the surgical scar and the rest had complete relief of symptoms.

\section{Discussion}

\section{Relevance and importance of ultrasound for steroid injections}

Corticosteroid injection is a popular option for musculoskeletal disorders ever since their introduction in the 1950s [4].

Table 2 Figure showing the VAS, PRWE and VAS scores for patients undergoing surgical release and steroid injections

\begin{tabular}{|c|c|c|c|c|c|c|}
\hline \multirow[t]{2}{*}{ Follow up } & \multicolumn{2}{|l|}{ DASH } & \multicolumn{2}{|l|}{ PRWE } & \multicolumn{2}{|l|}{ VAS } \\
\hline & Surgical release & Steroid injection & Surgical Release & Steroid injection & Surgical release & Steroid injection \\
\hline On first consultation & $82.2 \pm 11.4$ & $81.7 \pm 9.5$ & $81.5 \pm 6.2$ & $79.3 \pm 7.9$ & $6.7 \pm 1.2$ & $6.8 \pm 1.2$ \\
\hline $\begin{array}{l}3 \text { weeks (conserva- } \\
\text { tive } \mathrm{Mx} \text { ) }\end{array}$ & $76.7 \pm 12.15$ & $73.2 \pm 14.7$ & $79.6 \pm 9.0$ & $75.1 \pm 10.9$ & $4.9 \pm 1.8$ & $4.8 \pm 1.6$ \\
\hline 6 weeks & $5.5 \pm 2.7$ & $6.6 \pm 2.7$ & $11.9 \pm 5.4$ & $9.1 \pm 6.5$ & $2.2 \pm 1.1$ & $2.6 \pm 1.3$ \\
\hline 6 months & $1.7 \pm 1.9(P<0.05)$ & $1.0 \pm 1.6(P<0.05)$ & $3.4 \pm 2.6(P<0.05)$ & $1.7 \pm 2.1(P<0.05)$ & $1.0 \pm .0(P<0.05)$ & $1.0 \pm .0(P<0.05)$ \\
\hline
\end{tabular}


RCTs and systematic review of RCTs in the last two decades have consistently shown their benefit in de Quervain's tenosynovitis $[1,4,12,13]$. Rowland et al. did a systematic review, which included Five RCTs with 142 patients receiving corticosteroid injection with an overall follow-up period of 5.6 months (1.5-12 months) [13]. They showed resolution rates of symptoms higher than the controls (2.59 vs. $5.37,(p=0.05))$. The steroid group demonstrated better pain relief as measured by the visual analogue scale (VAS) and improvement in Dutch AIMS-HFF and DASH scores that were statistically significant [13]. Our study confirms the benefit observed with steroids but we additionally used the advantage of an ultrasound-guided procedure. We conducted this study to test our hypothesis that USG guided steroid injection is equally effective as surgery. We observed that USG guided steroid injections are comparable to surgical release in terms of pain relief, functional outcome, complications.

Blind steroid injection has a high chance of missing the separate compartment for EPB and hence leading to relapse or incomplete relief. Ultrasound has been used as a key tool for the diagnosis of de Quervain's tenosynovitis and more importantly for subtle anatomical variations like separate compartments for APL and EPB. Additionally, its use to deliver steroids when compared to blind injection has significantly improved outcomes as shown in some of the reports [21-26] (Table 3). In a series of 50 cadavers, Leversedge et al. showed an accuracy rate of $94 \%$ in the diagnosing presence of sub-compartments within the first compartment using USG. Their USG -guided injection accuracy was $100 \%$ and EPB compartment injection 96\% [24]. Another study, by Kang et al. in a series of 15 fresh cadavers, demonstrated not only a higher accuracy for USG guided injections (93.3\% vs. $40 \%$ ) to target the first extensor compartment but also better results when separate septum was present $(85.7 \%$ vs. $16.7 \%$ ) [30]. In a cadaver model involving 43 specimens comparing USG and anatomical landmark (AL) guided injection, Kutsikovich et al. showed that both techniques had an infiltration rate of 100\% [32]. In the presence of a septum, the USG group achieved a higher infiltration rate in EPB sub-compartments of 75\% accuracy in reaching the sub-compartment when compared to $33 \%$ in AL. Although statistically unconfirmed, they stated that USG aided injections in the presence of a septum [32]. In our series, more than $50 \%$ of patients had a separate sub-compartment and none of them came back with recurrence of symptoms, suggesting the effectiveness of USG guided injections.

Similar results were observed in clinical studies $[18,20$, $22,24]$. In a series of 43 wrists who were evaluated with USG, Kwon et al. showed a sensitivity of $100 \%$ and a specificity of $96 \%$ with a positive predictive value of $95 \%$ in diagnosing an intra-compartmental septum [20]. Kume et al. did an RCT involving 44 wrists comparing blind injections with
USG guided injections [18]. At 4 weeks, they reported a significant decrease in the VAS scores in the USG group from 80.3 to $25.6 \mathrm{~mm}(p=0.004)$ when compared to the manual group (from 78.0 to $58.2 \mathrm{~mm}(p=0.04)$ ) after injection [19]. Their results suggested that USG guided injections targeted the septation of EPB better than the blind injections [19]. However, there have been reports which have questioned the advantage of the use of USG including those with reports of increased chance of failure particularly with the presence of a sub-compartment [16, 23, 31, 32]. Nevertheless, its benefit and use are still advocated in view of a higher chance of placement of the needle at the pathological site [23, 31, 32]. In a level 1, study of randomized comparison between blind and USG guided injection involving 44 patients, Shin et al. observed sustained improvement in PRWE and pain scores at 4 and 12 weeks post-injection [16]. The authors observed that at 3 months, 10\% in USG showed recurrence of symptoms, which was marginally better than $26.3 \%$ in the blind group although this was statistically not significant [16]. In our study, we observed a benefit of USG-guided steroid injection after 6 months of follow up which was statistically significant. Sustained benefit of injections was seen in $93.7 \%$ of our patients. None of the patients with higher risks for recurrence, came back with relapse of symptoms. The major advantage of a USG guided procedure lies in its ability to diagnose the condition, checking for anatomical variability, while helping in counselling the patient on the prognosis of the condition [22]. Additionally, it helps in the accurate placement of the needle at the right pathological site [22]. The procedure is relatively inexpensive in our country costing \$15 in our institution and hardly takes a little more time than blind injections, which incidentally costs $\$ 10$. In the two patients with recurrence that was observed in our series after 6 months, we observed a common compartment for APL and EPB. In one of the patients who was a computer professional, we were unable to find the exact etiology. In the other, a homemaker, the soft tissue injury may have independently triggered a new episode. Incidentally, no studies have established an association between hand usage at work or trauma with this condition [33].

\section{Functional outcomes}

The literature continues to report complications associated with surgery like wound infections, delayed wound healing, radial nerve injury, scar hypertrophy and tenderness [27, 34]. In this regard, the role of USG guided steroid injections becomes important as recent reports suggest equally impressive results with them as well [19, 21-26]. Hence we aimed to address the question of whether USG guided injections offers a success rate comparable to surgery apart from its obvious advantage of saving time, cost and logistics for both patients, surgeons and insurance. There are no studies 


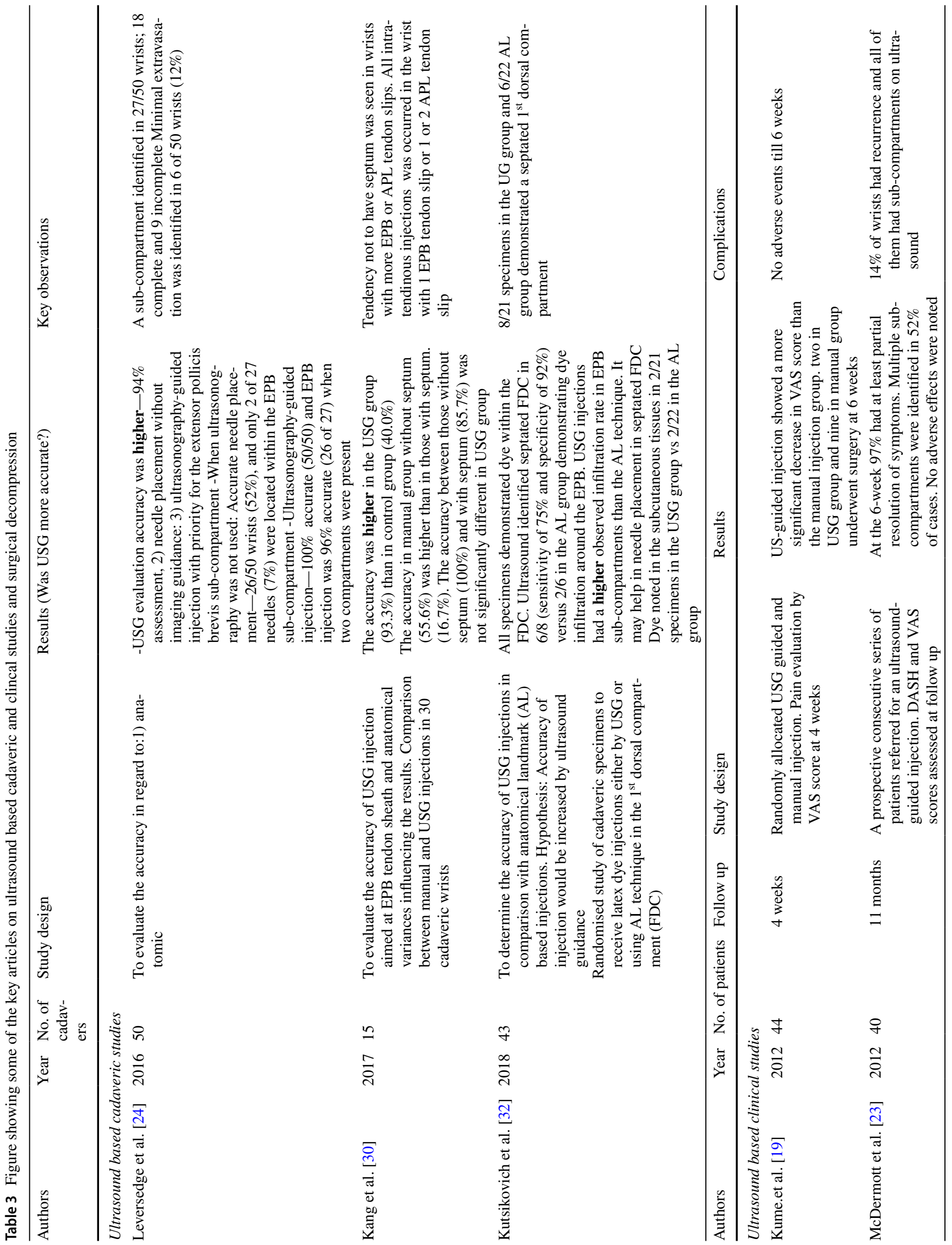




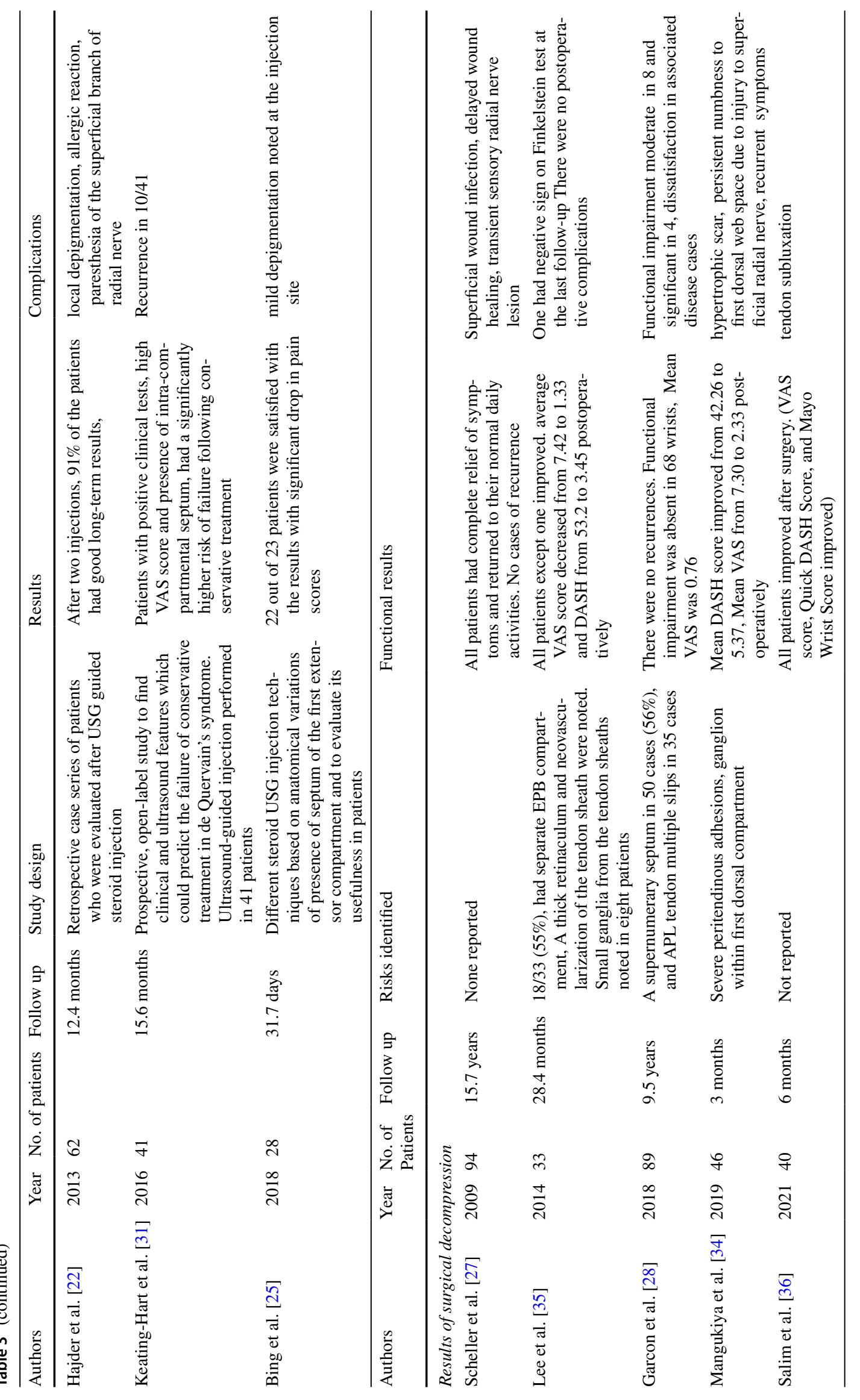


on comparison between the above two extremely effective procedures. Our study fills this information gap with a unique study comparing the two with respect to functional outcomes, recurrence and complications over a period of 6 months follow-up with a further assessment for recurrence for a maximum of 2 years.

Surgical release of the first extensor compartment has been the gold standard for de Quervain's tenosynovitis [34]. Long-term follow-up studies have shown impressive results with no reports of recurrence [27, 28] (Table 3). In a series involving 94 patients undergoing surgical release with an average follow-up of 15.7 years, Scheller et al. achieved complete relief of symptoms and normal return to daily activities in all their patients [27]. Garcon et al. also reported no recurrence and a satisfaction rate of $97.5 \%$ in a series of 89 patients with follow-up of 9.5 years [28]. Our results of surgery confirm similar outcomes with no recurrences and improvements in functional outcome scores that were statistically significant. We noted that patients who underwent surgical release for de Quervain's tenosynovitis had significant relief even on the first follow-up after surgery, and the same was maintained until the final follow-up. The total percentage change in DASH scores, PRWE scores and VAS scores at 6 months was also significant. This suggests that surgery is an effective treatment for de Quervain's tenosynovitis.

However, in our study, USG guided steroid injection provided equally rewarding results as surgery did. We observed significant and sustained relief in the patients treated with USG guided steroid injection and the results are comparable with other studies done for the same [16, 19, 21-29, 29, 30, 30, 31, 31, 32, 32-39]. Steroid injections when given under USG guidance can yield results, which are as good as surgery and avoid complications related to surgery and blind injections.

\section{Recurrence and complications}

Most of the studies noted good relief in the first 4-6 weeks of follow-up after which relapse of symptoms may be observed [10, 11, 13, 14, 16, 23, 31, 37]. However, the pattern of relief and the time of recurrence with the need for a second injection remains variable [9]. Majority of the studies have reported recurrence by $6-12$ weeks $[9$, 14, 18, 20, 30, 37] (Table 3). Earp et al. have reported relief in more than half among a series of 50 consecutive patients who were followed up over a year after a singleblind steroid injection for de Quervain's tenosynovitis [29]. In their opinion, recurrence wase observed within the first 6 months of follow-up [29]. These recurrences are significantly lower at 6 months whenever USG guided injections were used as nearly $3 / 4$ th of patients showed significant relief in other series [22, 23, 29, 31]. Even with USG guided injections, recurrences were observed more frequently in the presence of a separate septum [22, 31]. In one report, Hajdar et al. achieved $73 \%$ success after single USG guided injections with all recurrences being observed by 9 months similar to that of our patients [22]. Similarly, Keating-Hart et al. achieved $91 \%$ success initially, which was sustained at $76 \%$ at the end of the year with five patients each showing recurrence in the initial 6 weeks and later half, respectively [31]. Considering this fact, USG becomes an advantageous tool to counsel our patients on the prognosis of the condition while giving the injections. None of our patients required the need for a surgery even after 1 year of follow-up except two, which justifies the use of USG guided injections ahead of surgery. Literature has noted problems of hypopigmentation, flare reaction, radial nerve symptoms, thrombophlebitis, and weakness of grip strength with steroid injections $[1,10,16]$ (Table 3 ). We did not observe any such adverse reactions in our study.

Incidentally, our patients did not have any significant complications in surgery also, other than minor sensory disturbances at the affected site.

The strength of our study lies in achieving adequate matching of demographics from a similar pool of population while pursuing treatment during the same time period. We have used the DASH, PRWE and VAS scores for the outcome analysis as each of these scores have been shown to correlate with each other well and has also been used for assessment of function following both surgery and steroid injections in past studies [16, 38].

Limitations of our study lie in our inability to provide accuracy rates of diagnostic USG for comparison with therapeutic procedure findings as the injections, imaging evaluations and surgery were all done by different consultants. Although it was not the aim, functional scores at 1-year follow-up would have added value to our study. As expected, most of our patients' visits were untimely and irregular after 6 months, which is the case in most clinical conditions where patients get optimum relief. Hence, other than features of recurrence, functional scores would not have been useful for comparison. We have not done a detailed evaluation after 6 months, as this was not the aim of the study and have only evaluated for recurrence due to which we were unable to provide long-term followup results. However, recurrences are far much lesser after 6 months as reported in literature [29, 37].

Based on our observations, perhaps future studies could involve comparing the role of site and type of needle placement based on USG findings with various combinations of steroids along with hyaluronic acid preparations [25, 26]. The advent of WALANT technique for de Quervain's and its benefits in recent RCTs opens up its utility for comparison with USG guided techniques as well [39]. 


\section{Conclusion}

We observed that USG guided steroid injections are comparable to surgical release in terms of pain relief and functional outcome and with far less cost, complications and hospital stay. Surgical release is the gold standard treatment of de Quervain's tenosynovitis at present and we suggest that USG guided steroid injection can equal surgical results. The ultrasound findings preoperatively also help in surgical planning in correlating the number of APL tendons and separate compartment of EPB which prepares the surgeon to plan well both for injections and counseling of patients for prognostication.

Authors' contributions Conceptualization: $[\mathrm{AKB}]$; Methodology: [RV, $\mathrm{AKB}, \mathrm{KVR}]$; Formal analysis and investigation: [RV]; Writing-original draft preparation: [RV, AA]; Writing - review and editing: [AA, $\mathrm{AKB}]$; Funding acquisition: [AKB, RV]; Resources: [AKB]; Supervision: $[\mathrm{AKB}, \mathrm{AA}]$.

Funding Open access funding provided by Manipal Academy of Higher Education, Manipal.

\section{Declarations}

Conflict of interest All the authors declare no conflict of interest in this study.

Open Access This article is licensed under a Creative Commons Attribution 4.0 International License, which permits use, sharing, adaptation, distribution and reproduction in any medium or format, as long as you give appropriate credit to the original author(s) and the source, provide a link to the Creative Commons licence, and indicate if changes were made. The images or other third party material in this article are included in the article's Creative Commons licence, unless indicated otherwise in a credit line to the material. If material is not included in the article's Creative Commons licence and your intended use is not permitted by statutory regulation or exceeds the permitted use, you will need to obtain permission directly from the copyright holder. To view a copy of this licence, visit http://creativecommons.org/licenses/by/4.0/.

\section{References}

1. Ashraf MO, Devadoss VG (2014) Systematic review and metaanalysis on steroid injection therapy for de Quervain's tenosynovitis in adults. Eur J Orthop Surg Traumatol 24(2):149-157. https:// doi.org/10.1007/s00590-012-1164-z

2. Kutsumi K, Amadio PC, Zhao C et al (2005) Gliding resistance of the extensor pollicis brevis tendon and abductor pollicis longus tendon within the first dorsal compartment in fixed wrist positions. J OrthopRes 23(2):243-248

3. Peters-Veluthamaningal C, Winters JC, Groenier KH, MeyboomDeJong B (2009) Randomised controlled trial of local corticosteroid injections for de Quervain's tenosynovitis in general practice. BMC Musculoskelet Disord 10:131. https://doi.org/10.1186/ 1471-2474-10-131
4. Peters-Veluthamaningal C, van der Windt DA, Winters JC, Meyboom-de JB (2009) Corticosteroid injection for de Quervain's tenosynovitis. Cochrane Database Syst Rev. https://doi.org/10. 1002/14651858.CD005616.pub2

5. Kuo YL, Hsu CC, Kuo LC, Wu PT, Shao CJ, Wu KC, Wu TT, Jou IM (2015) Inflammation is present in de Quervain disease-correlation study between biochemical and histopathological evaluation. Ann Plast Surg 74(Suppl 2):S146-51. https://doi.org/10. 1097/SAP.0000000000000459 (PMID: 25650747)

6. Lee ZH, Stranix JT, Anzai L, Sharma S (2017) Surgical anatomy of the first extensor compartment: a systematic review and comparison of normal cadavers versus de Quervain syndrome patients. J Plast Reconstr Aesthet Surg 70(1):127-131. https://doi.org/10. 1016/j.bjps.2016.08.020

7. Hiranuma A, Houjo H, Sakaguchi S (1972) de Quervain's tenosynovitis and anatomical variation of first extensor compartment. Orthop Surg 23:1186-1188

8. Jackson WT, Viegas SF, Coon TM, Stimpson KD, Frogameni AD, Simpson JM (1986) Anatomical variations in the first extensor compartment of the wrist. A clinical and anatomical study. J Bone Jt Surg Am 68(6):923-6

9. Leslie BM, Ericson WB, Morehead JR (1990) Incidence of a septum within the first dorsal compartment of the wrist. J Hand Surg Am 15(1):88-91

10. Oh JK, Messing S, Hyrien O, Hammert WC (2017) Effectiveness of corticosteroid injections for treatment of de Quervain's tenosynovitis. Hand (N Y) 12(4):357-361. https://doi.org/10.1177/ 1558944716681976

11. Pensak MJ, Bayron J, Wolf JM (2013) Current treatment of de Quervain tendinopathy. J Hand Surg Am 38(11):2247-2250. https://doi.org/10.1016/j.jhsa.2013.06.003

12. Peters-Veluthamaningal C, Winters JC, Groenier KH, MeyboomDe JB (2009) Randomised controlled trial of local corticosteroid injections for de Quervain's tenosynovitis in general practice. BMC Musculoskelet Disord 10:131. https://doi.org/10.1186/ 1471-2474-10-131

13. Rowland P, Phelan N, Gardiner S, Linton KN, Galvin R (2015) The effectiveness of corticosteroid injection for de Quervain's stenosing tenosynovitis (DQST): a systematic review and metaanalysis. Open Orthop J 9:437-444

14. Mirzanli C, Ozturk K, Esenyel C, Ayanoglu S, Imren Y, Aliustaoglu S (2012) Accuracy of intrasheath injection techniques for de Quervain's disease: a cadaveric study. J Hand Surg Eur Vol 37(2):155-160

15. Zingas C, Failla JM, Van Holsbeeck M (1998) Injection accuracy and clinical relief of de Quervain's tendinitis. J Hand Surg Am 23(1):89-96

16. Shin YH, Choi SW, Kim JK (2020) Prospective randomized comparison of ultrasonography-guided and blind corticosteroid injection for de Quervain's disease. Orthop Traumatol Surg Res 106(2):301-306. https://doi.org/10.1016/j.otsr.2019.11.015

17. Nguyen ML, Jones NF (2012) Rupture of both the abductor pollicis longus and extensor pollicis brevis tendons after steroid injection for de quervain tenosynovitis. Plast Reconstr Surg 129(5):883e-886e

18. Yuen A, Coombs CJ (2006) Abductor pollicis longus tendon rupture in de Quervain's disease. J Hand Surg Eur 31(1):72-75

19. Kume K, Amano K, Yamada S, Kuwaba N, Ohta H (2012) In de Quervain's with a separate EPB compartment, ultrasoundguided steroid injection is more effective than a clinical injection technique: a prospective open-label study. J Hand Sur Eur 37(6):523-527

20. Kwon BC, Choi S-J, Koh SH, Shin DJ, Baek GH (2010) Sonographic identification of the intracompartmental septum in de Quervain's disease. Clin Orthop Relat Res 468(8):2129-2134 
21. Jeyapalan K, Choudhary S (2009) Ultrasound-guided injection of triamcinolone and bupivacaine in the management of de Quervain's disease. Skeletal Radiol 38:1099-1103

22. Hajder E, de Jonge M, van der Horst C, Obdeijn M (2013) The role of ultrasound-guided triamcinolone injection in the treatment of de Quervain's disease: treatment and a diagnostic tool? Chirurgie de la Main 32(6):403-407

23. McDermott JD, Ilyas AM, Nazarian LN, Leinberry CF (2012) Ultrasound guided injections for de Quervain's tenosynovitis. Clin Orthop Relat Res 470(7):1925-1931

24. Leversedge FJ, Cotterell IH, Nickel B, Crosmer M, Richard M, Angermeier E (2016) Ultrasonography-guided de Quervain injection accuracy and anatomic considerations in a cadaver model. J Am Acad Orthop Surg 24(6):399-404

25. Bing JH, Choi SJ, Jung SM et al (2018) Ultrasound-guided steroid injection for the treatment of de Quervain's disease: an anatomybased approach. Skeletal Radiol 47(11):1483-1490. https://doi. org/10.1007/s00256-018-2958-9

26. Orlandi D, Corazza A, Fabbro E, Ferrero G, Sabino G, Serafini G, Silvestri E, Sconfienza LM (2015) Ultrasound-guided percutaneous injection to treat de Quervain's disease using three different techniques: a randomized controlled trial. Eur Radiol 25(5):1512-1519

27. Scheller A, Schuh R, Hönle W, Schuh A (2009) Long-term results of surgical release of de Quervain's stenosing tenosynovitis. Int Orthop 33(5):1301-1303

28. Garçon JJ, Charruau B, Marteau E, Laulan J, Bacle G (2018) Results of surgical treatment of de Quervain's tenosynovitis: 80 cases with a mean follow-up of 9.5 years. Orthop Traumatol Surg Res 104(6):893-896. https://doi.org/10.1016/j.otsr.2018.04.022

29. Earp BE, Han CH, Floyd WE, Rozental TD, Blazar PE (2015) De Quervain tendinopathy: survivorship and prognostic indicators of recurrence following a single corticosteroid injection. J Hand Surg Am 40(6):1161-1165

30. Kang JW, Park JW, Lee SH et al (2017) Ultrasound-guided injection for De Quervain's disease: accuracy and its influenceable anatomical variances in first extensor compartment of fresh cadaver wrists. J Orthop Sci 22(2):270-274. https://doi.org/10.1016/j.jos. 2016.11.013

31. Keating-Hart E, Touchais S, Kerjean Y, Ardouin L, Le Goff B (2016) Presence of an intracompartmental septum detected by ultrasound is associated with the failure of ultrasound-guided steroid injection in de Quervain's syndrome. J Hand Surg Eur 41(2):212-219

32. Kutsikovich J, Merrell G (2018) Accuracy of Injection Into the first dorsal compartment: a cadaveric ultrasound study. J Hand Surg Am 43(8):777.e1-777.e5. https://doi.org/10.1016/j.jhsa. 2018.01.020

33. Dunn JC, Polmear MM, Nesti LJ (2019) Dispelling the myth of work-related de Quervain's tenosynovitis. J Wrist Surg 8(2):90 92. https://doi.org/10.1055/s-0039-1677741

34. Mangukiya HJ, Kale A, Mahajan NP, Ramteke U, Manna J (2019) Functional outcome of de Quervain's tenosynovitis with longitudinal incision in surgically treated patients. Musculoskelet Surg 103(3):269-273. https://doi.org/10.1007/s12306-018-0585-1

35. Lee HJ, Kim PT, Aminata IW, Hong HP, Yoon JP, Jeon IH (2014) Surgical release of the first extensor compartment for refractory de Quervain's tenosynovitis: surgical findings and functional evaluation using DASH scores. Clin Orthop Surg 6(4):405-409. https:// doi.org/10.4055/cios.2014.6.4.405

36. Salim B, Ansari MT, Kumar VS, Goyal A, Malhotra R (2021) Is pulley reconstruction better than pulley release for de Quervain's tenosynovitis? A double-blind randomized controlled trial. J Wrist Surg 10(5):377-384. https://doi.org/10.1055/s-0041-1725171

37. Witt J, Pess G, Gelberman RH (1991) Treatment of de Quervain tenosynovitis. A prospective study of the results of injection of steroids and immobilization in a splint. J Bone Joint Surg Am 73(2):219-222

38. Mehta SP, MacDermid JC, Richardson J, MacIntyre NJ, Grewal $\mathrm{R}$ (2015) A systematic review of the measurement properties of the patient-rated wrist evaluation. J Orthop Sports Phys Ther 45(4):289-298. https://doi.org/10.2519/jospt.2015.5236

39. Lee SK, Kim SG, Choy WS (2020) A randomized controlled trial of minor hand surgeries comparing wide awake local anesthesia no tourniquet and local anesthesia with tourniquet. Orthop Traumatol Surg Res 106:1645-1651

Publisher's Note Springer Nature remains neutral with regard to jurisdictional claims in published maps and institutional affiliations. 